\title{
Titrated intravenous opioids from the same syringe: an infection risk?
}

\author{
Monika Taylor, Jodie Bourke, Mark Anderson, Richard Davey, Anne-Maree Kelly, Byron \\ Guthrie
}

\begin{abstract}
Objective-(1) To compare the rate of contamination of syringes prepared under laminar flow conditions in pharmacy with those prepared by nurses in the emergency department; (2) to determine whether the time elapsed since preparation or number of doses given affected the contamination rate; (3) to determine whether any adverse effects resulted from bacterially contaminated drugs.

Methods-Prospective, blinded trial exploring the effect of method of preparation, time since preparation, and number of doses given on contamination rates and infective adverse events associated with bacterially contaminated specimens.

Results-The rate of bacterial contamination was $12 \%$ (95\% confidence interval $6 \%$ to $18 \%$ ). There was no difference in contamination rate in respect of method of preparation, number of doses given, or time since preparation. No infective complications were identified.

Conclusions-Abandonment of titrated intravenous opioids is not justified by the results. However, there is concern about the use of this technique of pain control for immunocompromised patients and those with prosthetic heart valves. (F Accid Emerg Med 1997;14:33-35)
\end{abstract}

Keywords: opioid; intravenous; contaminants; immunosuppression

Western Hospital, Footscray, Australia: Department of Emergency Medicine M Taylor J Bourke

A-M Kelly

Department of

Pathology

$M$ Anderson

R Davey

Pharmacy

Department

B Guthrie

Correspondence to: Dr A M Kelly, Emergency Department, Western Hospital Footscray, Private Bag, Footscray 3011, Australia

Accepted for publication 5 September 1996
The administration of titrated intravenous opioids is a common method of analgesia in emergency departments. This method is also used in other critical care areas such as coronary and intensive care units, as well as in anaesthesia and ambulance services.

Although licensed for use as a single dose, the contents of ampoules of morphine and pethidine are often used more than once for the same patient in the context of titrated intravenous opioid administration for pain control. Presently, neither the opioid ampoules nor the fluids used to dilute them contain bacteriostatic or bacteriocidal agents. Thus if multiple doses were to be given from a syringe over time and contamination of the fluid occurred, there could be an opportunity for infection. In usual practice these preparations are prepared by nursing staff at the time of use. A pilot study ${ }^{1}$ has shown that contamination of these preparations occurs. There is no other published research on this aspect of practice.
The aims of this study were: (1) to compare the rate of contamination of syringes prepared under laminar flow conditions in pharmacy with those prepared by nurses in the emergency department; (2) to determine whether the time elapsed since preparation or number of doses given effected the contamination rate; and (3) to determine whether any adverse effects resulted from bacterially contaminated drugs.

\section{Methods}

A prospective, blinded, comparison trial comparing contamination rates in specimens of opioid used for titrated analgesia was conducted between 20 March and 27 September, 1995 at Western Hospital Footscray, a 300 bed teaching hospital. Only narcotic preparations used during the morning shift where eligible for inclusion to ensure that the study specimens were delivered to the microbiology department for culture without lengthy delay.

\section{SYRINGE PREPARATION}

Pethidine and morphine used in the emergency department for titrated intravenous opioid administration were studied. During the study syringes were prepared on alternate weeks in advance by pharmacy department staff or at the time of use by emergency department nursing staff. Nursing staff prepared the drugs using standard "clean" technique; pharmacy employed aseptic techniques and worked within a laminar flow cabinet. The opioid was routinely diluted into a final volume of $10 \mathrm{ml}$ with normal saline. Staff administering the drug were not blinded to the method of preparation.

\section{SYRINGE USE}

After each administration to the patient by nursing staff, the needle was replaced with a new, sterile, guard covered needle. Each syringe, when used, was labelled with the patient's name. Used syringes in which some opioid preparation remained were forwarded to the Western Hospital microbiology department for processing. The length of time syringes had been in use and the number of times each had been used were recorded.

\section{SYRINGE TESTING}

Using aseptic technique the first millilitre of fluid from each syringe was transferred to a horse blood agar plate. The second millilitre of remaining fluid was transferred to a cooked meat broth bottle, taking care to insert the 
Table 1 Bacteria cultured from opioid fluid

\begin{tabular}{ll}
\hline Isolate & No of syringes \\
\hline Coagulase negative staphylococcus (CNS) & 9 \\
Micrococcus & 1 \\
CNS and $\alpha$ haemolytic streptococcus & 1 \\
Non-haemolytic streptococcus and & 1 \\
$\quad$ diphtheroids & \\
\hline
\end{tabular}

needle, and the needle only, into the broth. Plates were incubated for two days and broth bottles for one week. Colonies seen on plates were enumerated and identified. Growth noted in bottles was plated and subsequently identified. Bottles which appeared to be sterile were subcultured after one week, aerobically and anaerobically, and any growth subsequently identified. The microbiology department was blinded with respect to the method of preparation of the sample, the number of uses, and the elapsed time since first usage.

QUALITY ASSURANCE

In order to ensure that any isolates found were not contaminants introduced in the laboratory, 60 syringes were aseptically prepared within the laboratory using normal saline and were subjected to the same culture procedures. This method was considered most valid as the step of mixing the drugs is a potential opportunity for contamination.

\section{CLINICAL ASSESSMENT}

In cases where organisms were grown, the patients' histories were examined for any evidence of infection at an intravenous access site or of subsequent systemic infection. Their primary diagnosis was also noted.

\section{STATISTICAL PROCESSING}

The Stata package was used to process the data, ${ }^{2} \chi^{2}$ and Fisher's exact tests being used where appropriate.

\section{Results}

Of 100 syringes tested, growth was noted from $12(12 \%, 95 \%$ confidence interval $6 \%$ to $18 \%)$. Five were positive by plate culture and seven initially by broth culture. The isolates identified are shown in table 1.

Of the 12 syringes yielding growth of microorganisms, six had been prepared in the emergency department and six in the pharmacy department.

Neither duration since first usage nor the number of times used correlated with contamination rate.

The 60 syringes prepared in the microbiology laboratory produced one positive culture, a coagulase negative staphylococcus growth in broth. Compared with the 12 isolates in 100

Table 2 Outcome of patients associated with syringes yielding bacterial growth

\begin{tabular}{llll}
\hline Diagnosis & No & IV site phlebitis & Systemic infection \\
\hline Myocardial infarction & 2 & 0 & 1 -Urinary tract infection \\
Angina & 2 & 0 & 1 -Urinary tract infection \\
?Pulmonary embolism & 1 & 0 & 0 \\
Renal colic & 2 & 0 & 0 \\
Gastrointestinal disease & 3 & 0 & 1 -Appendicitis \\
Musculoskeletal & 2 & 0 & 0 \\
\hline
\end{tabular}

cases from patients this difference is statistically significant (Fisher's exact test, $P=0.03$ ).

Clinical details of the patients from whom positive cultures were obtained are shown in table 2 .

\section{Discussion}

SOURCE OF CONTAMINATION

The preliminary study ${ }^{1}$ from which this work proceeds found $13.5 \%$ of syringes used in a similar fashion grew microorganisms. A similar array of microorganisms was identified. In the current study, the contamination rate was $12 \%$, statistically indistinguishable from the pilot study results $\left(\chi^{2}, \mathrm{P}=0.77\right)$.

As exactly half of the contaminated syringes came from each source, this study shows that there is little to be gained from preparing opioid dilutions in an aseptically enhanced environment such as the pharmacy department.

The laboratory quality control limb of the study shows that the laboratory itself was not the source of the organisms cultured.

The finding that neither the time since first usage nor the number of usages is relevant is interesting and somewhat unexpected. The likely explanation is that contamination is a random event, without a direct relation to either of these variables.

Since both syringe preparation and laboratory assessment have been excluded as contaminant sources and there is no correlation with usage time or frequency, it would appear that microorganisms enter the opioid fluids in the syringes during their use. These data suggest that, in spite of the procedures aimed at asepsis, there will always be a potential for fluid contamination, arising solely from clinical use, of the order of $12 \%$.

\section{THE CLINICAL OUTCOME}

In this series there was no evidence that bacterial contamination of opioid fluid contributed to patient morbidity or mortality. The systemic infections noted were either the presenting complaint per se (appendicitis) or incidental (urinary tract infections). One intravenous access site "tissued" and the cannula was subsequently removed, but phlebitis was not noted.

In no case was the organism grown from the opioid fluid the same as that responsible for a pre-existing systemic infection in the patient.

\section{CLINICAL CONSEQUENCES}

We have shown that opioid analgesic fluids used in a titrated manner do not remain sterile. As many as $18 \%$ of syringes might - and in this series $12 \%$ did-become contaminated with skin commensal flora. These microorganisms might not challenge healthy individuals and indeed no adverse events were observed. However, were they to be injected into the blood stream of immunocompromised or immunosuppressed patients, local or disseminated sepsis would be possible. Other people at potential risk are those with internal prostheses, in particular prosthetic heart valves. 
It might be suggested that, because it has been shown that opioid fluids given in the manner described become contaminated with bacteria at a rate of approximately $12 \%$, the use of this method of analgesia should be abandoned. However, titrated intravenous analgesia has significant benefits over alternative methods which must be considered. In particular, onset of analgesia is rapid and the dose can be titrated to the needs of the patient. As no adverse event attributable to contamination was found, we believe that a total abandonment of this practice is not warranted. However, when treating immunosuppressed or immunocompromised patients or those with prostheses, the risk of the procedure must be weighed against the potential benefit.

Alternative techniques to reduce the contamination risk of opioid solutions include the addition of bacteriocidal agents to opioid solutions or the use of closed system infusions. Unfortunately, the equipment requirements of the latter technique would make its implementation in emergency departments difficult.

The use of opioids in this manner is not limited to the emergency department. Titrated intravenous opioids are also used by some ambulance services, coronary and intensive care units, and during anaesthesia. The relative risk of an infection to the patient given contaminated opioid fluid may differ in these settings as the types of patients and their immunocompetence are different.
There are other drugs used in a similar manner (that is, titrated to effect, with more than one dose being given from a preprepared syringe) that do not contain bacteriostatic or bacteriocidal agents. These include midazolam, vecuronium, thiopentone, propofol, atracurium, and fentanyl. It is possible that these solutions also become contaminated. No research into this subject has been published.

\section{CONCLUSION}

The results of this study indicate that despite careful preparation and handling the fluid in $12 \%$ of syringes used to give titrated opioid analgesic became contaminated with bacteria. Although no adverse consequence was identified in the patients in this study, the potential for infective complications remains. Abandonment of this practice is not justified by our results; however, there is concern about the use of this technique of pain control for immunocompromised patients and those with prosthetic heart valves.

We would like to thank Liz Edmonds, Linda Cotone, and the staff of the pharmacy and emergency departments for their assistance with this project.

1 Kelly AM, Davey R, Guthrie B, Anderson M, Taylor M, Bourke J, et al. Are titrated intravenous opioids an infection risk? Emerg Med 1995; 7:117.

2 Computing Resource Centre. Stata reference manual: release 3, 5th ed. Santa Monica, CA, 1992. 